\title{
Strategies for an HIV vaccine
}

\author{
Norman L. Letvin \\ Harvard Medical School, Beth Israel Deaconess Medical Center, 41 Avenue Louis Pasteur, RE-113, Boston, Massachusetts 02215, USA. \\ Phone: (617) 667-2042; Fax: (617) 667-8210; E-mail: nletvin@caregroup.harvard.edu.
}

J. Clin. Invest. 109:15-20 (2002). doi:10.1172/JCI200215985.

The development of an HIV vaccine poses an unprecedented challenge to the scientific community. The inexorable spread of HIV worldwide and the devastating clinical consequences of AIDS can only be contained by an effective vaccine. Yet, almost two decades after the first demonstration of HIV and its etiologic role in AIDS, this vaccine still is a goal, not a reality. In this Perspective, I consider the unique problems for vaccine development posed by the biology of HIV infection, and I summarize recent advances in our understanding of the immune control of HIV and the implications of these advances for vaccine development. After reviewing the sobering history of failed traditional vaccine strategies, I discuss the rationale for vaccines with novel designs and their experimental successes to date.

\section{HIV biology}

HIV offers a uniquely difficult target for vaccine development. The HIV isolates that infect humans and cause AIDS include a genetically diverse population of viruses (1). The HIV responsible for causing AIDS in much of West Africa is referred to as HIV-2; the HIV that causes AIDS throughout the rest of the world is referred to as HIV-1. HIV-2 and HIV-1 are so divergent in their genetic sequences that their envelope glycoproteins are often not immunologically cross-reactive. Moreover, the viruses of the HIV-1 group include disparate viral clades or subtypes that are clustered epidemiologically in distinct geographic regions. These various clades of HIV-1 isolates differ from one another so dramatically in their genetic sequences, and therefore their antigenic characteristics, that it has been suggested that different geographic regions of the world may actually require different vaccines. The parallel development of various region-specific HIV vaccines would clearly be both difficult and time-consuming.

Genetic diversity is also continuously generated in the course of an HIV infection in a single infected individual, as the inaccurate enzymatic machinery of this virus's replication results in ongoing production of mutant virions. This process engenders such a genetically heterogeneous population of virions that an antibody that can neutralize one HIV isolate may fail to neutralize another from the same individual. Such an extraordinary degree of genetic diversity among HIV isolates immeasurably complicates the process of HIV vaccine development.

Other aspects of the biology of HIV infections also have substantial implications for AIDS vaccine development. HIV is transmitted both venereally and hematogenously. Therefore, there is reason to suppose that an effective HIV vaccine must elicit both mucosal immunity, to contain sexually transmitted virus, and systemic immunity, to contain virus transmitted directly into the bloodstream. Furthermore, HIV is likely transmitted both as cell-free and as cell-associated virus. Therefore, more than a single type of immunity must be elicited by a vaccine if that vaccine is to be effective. Cell-free virions can be bound and neutralized by antibody, while cell-associated virus can be eliminated by cell-mediated immune responses. Finally, and most troubling for the prospects of developing an effective HIV vaccine, infection with this virus universally results in high levels of viral replication that persist in the face of seemingly robust anti-viral antibody and cell-mediated immune responses. Unlike most other viral infections in humans, replicating HIV is never fully cleared. Moreover, the level and persistence of viral replication are inexorably tied to the pathogenicity of the virus, with high persistent HIV replication being associated with rapid progression of clinical disease. The universal persistence of viral replication in spite of potent immune responses raises the specter that no vaccine-elicited immune response may be capable of fully eliminating or containing indefinitely the replication of HIV.

\section{AIDS animal models}

Appropriate animal models have been key to the elucidation of AIDS immunopathogenesis and the assessment of HIV vaccine strategies. Nonhuman primates represent a particularly powerful model for the study of AIDS. The HIV-1 and HIV-2 isolates that infect humans are members of a large family of lentiviruses that endemically infect nonhuman primate species of Africa. These nonhuman primate viruses are known as simian immunodeficiency viruses (SIVs). Interestingly, they cause no disease in their natural host species. However, some of these isolates cause AIDS when inoculated experimentally in Asian macaques (2). Moreover, chimeric viruses have been developed that 
express HIV-1 envelope glycoproteins on SIV backbones. Some of these simian human immunodeficiency viruses (SHIVs) can cause rapidly progressive AIDS-like disease in macaques (3).

\section{AIDS immunopathogenesis}

Many acute viral infections are cleared by neutralizing antibodies induced by the replicating viruses. These antibodies bind to viral particles and block the ability of the particles to attach to and subsequently infect cells. Our emerging understanding of antibody responses in HIV-infected individuals suggests that such immune responses are not likely to be critical in blocking HIV spread. While high-titer anti-HIV antibodies certainly develop in infected individuals, these antibodies display only weak HIV-neutralizing activity. Moreover, the partial containment of replicating HIV usually seen during the first weeks following initial infection precedes the development of antibodies that can neutralize the virus. These observations imply that the virus-specific antibody response does not play a critical role in either the chronic or early containment of HIV replication in the infected individual.

Nevertheless, there is reason to suppose that neutralizing antibodies will be important in the development of an effective HIV vaccine and that they can be elicited by immunization. A limited number of mAb's have been developed that neutralize diverse HIV-1 isolates, confirming that shared, neutralization-sensitive viral domains exist. Infection by cell-free virus can probably only be blocked immunologically by antibodies that target such viral epitopes. Moreover, studies in the SHIV/macaque model clearly indicate that passively administered neutralizing antibodies can prevent an AIDS virus infection if sufficiently high levels of circulating antibody are achieved (4). Thus, if such antibodies can be elicited through vaccination, they should be effective in blocking transmission of virus. It is problematic, however, that the neutralization-sensitive domains of the virus have proven poorly immunogenic. Configuring a subunit immunogen that can elicit an antibody response that neutralizes a diversity of HIV isolates stands as perhaps the greatest challenge facing HIV vaccine development at this time.

The immunologic mechanisms responsible for containing HIV replication are very different from those responsible for controlling many of the other viruses for which effective vaccines have been developed. These other viruses are contained primarily or solely by neutralizing antibody. HIV, on the other hand, appears to be controlled predominantly by cell-mediated immunity. Thus, soon after the indentification of HIV as the etiologic agent in AIDS, it was shown that $\mathrm{CD}^{+}$lymphocytes can inhibit HIV replication in $\mathrm{CD}^{+} \mathrm{T}$ cells in vitro (5). Later work showed that a virus-specific $\mathrm{CD}^{+} \mathrm{CTL}$ response precedes the early, partial control of HIV replication in acutely infected individuals (6). Moreover, the clinical status of chronically HIV-infected individuals is associated with the levels of circulating virus-specific $\mathrm{CD}^{+} \mathrm{CTLs}$, high levels being predictive of a stable immunologic function (7). In fact, newly developed, highly quantitative assays for detecting these cell populations have recently demonstrated extremely high levels of HIV-specific CTLs in both acutely and chronically infected individuals (8). A definitive and direct demonstration of the importance of $\mathrm{CD}^{+}$lymphocytes in the control of viral infection came from work in the SIV/macaque model, where monkeys depleted of $\mathrm{CD}^{+}$lymphocytes by mAb infusion and then infected with SIV never controlled early viral replication. These animals went on to die with a rapidly progressive AIDS-like disease (9). Taken together, these findings make a strong argument for the importance of CD8 ${ }^{+}$CTLs in controlling HIV replication, and they suggest that an effective HIV vaccine must elicit such an immune response.

Immune studies in normal mice and humans have clearly shown that $\mathrm{CD} 8^{+}$CTLs function normally only when optimal $\mathrm{CD} 4^{+} \mathrm{T}$ lymphocyte help is available to support CTL function. Hence, it is not surprising that control of HIV and stable clinical status have been shown to be associated with high levels of virus-specific $\mathrm{CD}^{+} \mathrm{T}$ lymphocyte help (10). An effective HIV vaccine would therefore be expected to elicit virus-specific $\mathrm{CD} 4^{+} \mathrm{T}$ lymphocyte help in addition to $\mathrm{CD} 8^{+} \mathrm{CTLs}$.

\section{Traditional vaccine designs}

The studies done to date to elucidate the replication of HIV and the immunopathogenesis of AIDS suggest that HIV is unique in its biology and may therefore not be amenable to control by immune responses elicited through traditional vaccine modalities. In fact, experiments in nonhuman primates and early-phase human studies bear out this supposition, providing convincing evidence that live attenuated virus vaccines, inactivated virus vaccines, and recombinant protein vaccines are all likely to be ineffective in preventing HIV infection and AIDS (Table 1).

Viruses can be attenuated in their in vivo pathogenicity by propagation in vitro, a process that generates limited numbers of mutations in the viruses. Because these viruses replicate in vivo and therefore elicit robust immune responses, infection with such pathogenically attenuated viruses represents an effective means of vaccinating humans to prevent measles, polio, and chicken pox. Preliminary studies in the $\mathrm{SIV} /$ macaque model suggested that viruses can be altered by molecular manipulation through the deletion of a limited amount of genetic material, and that such viruses become infectious but pathogenically
Table 1

Traditional designs for an HIV-1 vaccine

$$
\text { Design }
$$

Live, attenuated virus Inactivated viruses with adjuvants

Recombinant envelope protein

\section{Limitations}

Pathogenicity in vaccinees

Restricted specificity of neutralizing antibodies, absence of CTLs No neutralizing antibodies for patient isolates of HIV-1; absence of CTLs 
Table 2

Novel designs for an HIV-1 vaccine

\begin{tabular}{|c|c|}
\hline Design & Limitations \\
\hline Plasmid DNA & Limited immunogenicity in humans \\
\hline \multicolumn{2}{|l|}{ Live, recombinant vectors: } \\
\hline \multicolumn{2}{|l|}{ Pox viruses } \\
\hline Vaccinia & Dissemination in immunosuppressed vaccinees \\
\hline MVA, NYAC & Limited experience in humans \\
\hline Canary pox & Limited immunogenicity in humans at achievable dosages \\
\hline Gene-deleted adenovirus & Pre-existing immunity to adenovirus may limit immunogenicity \\
\hline alphaviruses, adeno-associated virus & Limited experience in humans \\
\hline Envelope subunit immunogens & No elicitation of neutralizing antibodies \\
\hline
\end{tabular}

attenuated. Moreover, these studies showed that prior infection with such attenuated viruses prevents subsequent infection with pathogenic wild-type virus (11). While reports of these findings raised hopes that a live attenuated HIV vaccine might be feasible, subsequent studies have shown that this approach to HIV vaccine design is flawed. Further work in the $\mathrm{SIV} /$ macaque model showed that newborn monkeys or adult monkeys infected for a long period of time with such vaccine strains of virus eventually develop AIDS and die (12). Similarly, a cluster of human infections has been described in which the HIV isolate was crippled in its replication competence and, accordingly, attenuated in its pathogenicity. However, as in the SIV/macaque studies, these infected individuals eventually went on to develop AIDS, albeit with a delay in time from infection to onset of disease. Such studies have suggested that it is unlikely to prove possible to uncouple the high level of replication of an attenuated AIDS virus needed to elicit protective immunity and the eventual pathogenicity of the virus. There is, therefore, little enthusiasm at this time for pursuing live attenuated virus strategies for an HIV vaccine.

While inactivated virus vaccine strategies have proven useful for preventing infections with influen$\mathrm{za}$ and polio virus in humans, they have been disappointing when assessed in the SIV/macaque model. This vaccine modality provided protection in monkeys that were challenged with SIV identical to the virus used in creating the vaccine, as well as in monkeys challenged at the time immunity was maximal (13). However, this vaccine protection proved neither broad nor robust. Thus, protection has not been demonstrated in this model when the strains of challenge virus and vaccine virus were even slightly disparate genetically or when the vaccinated monkeys were challenged even a few weeks after peak immunity was reached. Moreover, some studies suggested that the protection seen in this animal model may have reflected experimental artifact rather than virus-specific immunity (14). Nevertheless, inactivated virus immunogens were evaluated in limited early-phase human immunogenicity trials (15) with disappointing results. Such immunogens did not elicit antibody responses that neutralized HIV isolates, since the immunogen proved to have very little retained viral envelope glycoprotein. Moreover, since there was no synthesis of proteins in cells initiated by this immunogen, it did not induce CTL responses. Thus, as is the case for the live attenuated vaccine strategy, there seems to be little basis for pursuing further studies with inactivated HIV immunogens.

Finally, highly purified viral protein, produced through recombinant DNA technology, has been a highly effective immunogen for preventing hepatitis B virus infection in humans. Some years ago, this approach to HIV vaccine design was evaluated in nonhuman primate models, using recombinant HIV envelope glycoprotein as an immunogen. Animals enjoyed only modest protection, and only when the challenge virus and immunizing envelope glycoprotein were identical in sequence (16). Nevertheless, early-phase human immunogenicity trials have been carried out. Antibody responses elicited in these studies were modest in titer and very limited in the spectrum of HIV isolates that they neutralized. Moreover, as would be expected with a subunit immunogen, these proteins did not elicit CTL responses. Phase III efficacy trials are underway at this time with envelope subunit immunogens in both the US and Southeast Asia, supported by private sector resources, but there is little optimism in the scientific community that these studies will demonstrate meaningful protection against HIV infection, since the immune responses crucial for HIV containment will not be elicited by this approach. Thus, the traditional approaches have proved disappointing in the effort to create an effective HIV vaccine.

\section{Novel vaccine designs}

Recognition of the limitations of these traditional immunization strategies for preventing HIV infections has inspired researchers to explore a plethora of novel vaccine designs. The most promising of these approaches involve the use of plasmid DNA immunogens and live, recombinant vectors (Table 2).

It was shown more than a decade ago that plasmids encoding proteins under the control of potent promoters can be immunogenic if inoculated intramuscularly in small laboratory animals. Subsequent work has shown that this immunogenicity can be substantially enhanced through the delivery of these plasmids formulated with particular adjuvants or cytokines. Such immunogens have proven particularly useful in eliciting cell-mediated immune responses (17). Moreover, a number of studies have suggested that plasmid DNA is quite effective as a priming or initial immunogen in a bimodal vaccine strategy (18). While this approach provides a safe means of eliciting CTL responses, preliminary data in large animal studies 
have raised questions as to whether sufficiently large inocula of plasmid DNA can be administered in humans to elicit useful immune responses. Nevertheless, a number of early-phase human studies are planned and ongoing to explore plasmid DNA immunogens as HIV vaccine candidates.

Live recombinant vectors are also being explored as tools for eliciting immune responses against HIV. Genes of HIV can be inserted by molecular approaches into live, replication-competent microorganisms. The resulting recombinant microorganisms then can serve to carry these genes. Upon infection with these recombinant microorganisms, immunity is elicited to the vector and to the product of the HIV gene carried by that vector. Such immunogens have proven particularly useful for eliciting CTLs, since the HIV proteins are produced intracellularly by the replicating vector and therefore enter the MHC class I processing pathway. The microorganisms best studied as potential vaccine vectors are the pox family of viruses. The prototype member of this family is vaccinia, the replicationcompetent virus that served as the primary vaccine virus in the worldwide smallpox eradication campaign. Although studies in nonhuman primates have shown that recombinant vaccinia viruses can elicit potent CTL responses to HIV and SIV proteins (19), safety concerns have dampened enthusiasm for this vector as an HIV vaccine candidate. Vaccinia has been shown to disseminate in immunocompromised humans, sometimes causing a fatal encephalitis (20). Because an HIV vaccine is most desperately needed in regions of the world in which HIV infections are endemic, significant numbers of individuals already infected with HIV, and therefore immunosuppressed, would likely receive an AIDS vaccine during a campaign to immunize an entire population. A well-founded fear exists that a substantial number of those already HIV-infected individuals would develop a fatal vaccinia infection upon such a vaccination.

Other pox viruses have therefore received attention as potential HIV vaccine vectors. Perhaps the most interesting of these pox viruses is modified vaccinia Ankara (MVA). Generated from a parental vaccinia virus isolate by multiple in vitro passages, MVA carries a large number of deletions, leaving it infectious and immunogenic but highly attenuated in its pathogenicity. Nonhuman primate studies have shown that MVA elicits impressive immune responses, either as a stand-alone immunogen or as a boosting immunogen following plasmid DNA priming. Another similarly generated, gene-deleted vaccinia virus, referred to as NYVAC, has been shown in nonhuman primate studies to elicit an immune response comparable to that elicited by MVA when used as a vaccine vector. Both MVA and NYVAC will soon be evaluated as HIV vaccine vectors in early-phase human testing.

The most extensively studied of the pox viruses as potential HIV vaccine vectors are the avian pox viruses. Canary pox undergoes an abortive cycle of replication in human cells but initiates the synthesis of viral proteins during that process. Presumably, this level of protein expression is sufficient for MHC class I processing of the expressed proteins to occur. Recombinant canary pox constructs have undergone extensive human testing in recent years (21). These studies have shown recombinant canary pox to be safe and immunogenic, eliciting antibody responses in $70 \%$ of vaccinees and CTL responses detectable at any single point in time following vaccination in approximately $30 \%$ of individuals. An efficacy trial in Southeast Asia is currently being considered for a recombinant canary pox immunogen.

Perhaps the most promising of the live recombinant vectors assessed to date as a potential HIV vaccine is the gene-deleted adenovirus that was developed as a vector for gene therapy. The serotype 5 adenovirus, made replication-incompetent by deletion or inactivation of the E1 and E3 genes, has demonstrated impressive immunogenicity in both murine and nonhuman primate studies (22). These vectors have elicited both high-titer antibody and high-frequency CTL responses in these animal models. In fact, early-phase HIV immunogenicity trials with this vector are ongoing in humans. Preliminary findings in these trials have indicated that pre-existing antibody responses to adenovirus serotype 5 in humans who were previously infected with this common pathogen significantly dampen the in vivo expression, and therefore immunogenicity, of these vaccines, but a number of strategies are currently being considered to circumvent this prob$\mathrm{lem}$. The immunogenicity of these recombinant vectors is most impressive if they are used to boost plasmid DNA-primed immune responses. Adequate immune responses to HIV may therefore be elicitable with this vector system in adenovirus serotype 5-immune humans by priming them with plasmid DNA vaccines before immunizing them with the recombinant adenovirus serotype 5 constructs. It may also be possible to construct analogous vaccines with comparable immunogenicity using unusual serotype adenoviruses, isolates to which most humans have not been previously exposed. Alternatively, vaccine constructs might be developed using adenovirus isolates from nonhuman primate species. Such viruses are not natural human pathogens and therefore have not infected humans. Vectors constructed from such viruses should, however, prove immunogenic in humans.

A number of other replication-competent viruses are also being explored as potential vectors for HIV vaccines. These include single-strand RNA alphaviruses (Venezuelan equine encephalitis virus and Semliki forest virus) and the parvovirus adeno-associated virus. The bacterial vector systems that are receiving attention at this time include the attenuated Mycobacterium Bacille Calmette-Guerin and some of the pathogenically attenuated enteric bacteria. All of these approaches will be tested in the near future for immunogenicity in human vaccine trials.

Developing an immunogen that elicits anti-HIV envelope antibody responses that can neutralize a diversity of HIV isolates has been one of the most intractable problems that have arisen in the process 
of HIV vaccine development. A number of novel strategies are being actively pursued in an effort to generate such an envelope immunogen. Assuming that genetically conserved, neutralizing epitopes of HIV envelope may be shielded from the immune system during the process of infection with the virus, investigators are assessing immunogens constructed by removing $\mathrm{N}$-linked glycans and variable loop structures from the HIV envelope. Some have suggested that an effective vaccine might be created by constructing a subunit immunogen that conforms to the native folding of the HIV envelope glycoprotein. Since the envelope glycoproteins exist as oligomers on the native virus, attempts are being made to create a variety of stable oligomeric envelope proteins for evaluation as immunogens. Recent advances in our understanding of the process of HIV infection of a cell indicate that the envelope of the virus undergoes a series of stereotypic conformational changes during the process of viral fusion to the cell membrane. Attempts are being made to develop subunit immunogens that mimic such fusion intermediate forms of the virus envelope. Finally, since an effective HIV vaccine must elicit antibodies that bind to neutralizing determinants of a variety of HIV envelopes, some are arguing that polyvalent envelope mixtures should be assessed as potential immunogens. Incorporation of any such immunogen in a HIV vaccine would represent a dramatic departure from usual viral subunit vaccines.

Although the ultimate configuration of an effective HIV vaccine remains uncertain, there is a growing consensus that it will require more than a single vaccine modality. In a bimodal vaccine approach, a live recombinant vector or plasmid DNA could be used to elicit CTLs, while a subunit immunogen could be used to induce a neutralizing antibody response. Moreover, a growing body of evidence indicates that a high-frequency CTL response is best elicited by combining two complementing vaccine modalities. This has been most clearly demonstrated in studies combining plasmid DNA with live recombinant MVA or adenovirus immunizations. Thus, even a CTL-based vaccine is likely to make use of two distinct vaccine modalities as a prime/boost series of immunizations. Such an approach represents a radical change from traditional vaccine designs.

\section{Vaccination to prevent clinical disease}

We are accustomed to considering a particular viral vaccine effective if it blocks infections by that virus. A vaccine strategy that provides anything short of that ideal level of protection would be considered inadequate. There is, however, a shared conviction that for a vaccine to prevent HIV infections, the immunogen must elicit an antibody response that neutralizes a diversity of HIV isolates. Since we remain unable to elicit such an immune response with available vaccine prototypes, there has been a long-standing pessimism in the field of HIV research about the possibility of creating a useful HIV vaccine.
New findings in nonhuman primate studies have, however, suggested that available vaccine technologies may confer important benefits short of complete protection from infection. Monkeys vaccinated with a variety of novel immunogens, including plasmid DNA, cytokine-augmented plasmid DNA, recombinant MVA, recombinant gene-deleted adenovirus, and plasmid DNA followed by recombinant MVA or gene-deleted adenovirus $(18,22-25)$ show some improvement in their ability to control infection with highly pathogenic AIDS viruses. These vaccines did not elicit broadly neutralizing antibody responses and, accordingly, did not provide sterilizing immunity, but all of these vaccines elicited virus-specific CTL responses and conferred partial control of viral replication to vaccinated animals. Moreover, the level of viral replication in the animals correlated with the rapidity of disease progression. Those animals that demonstrated good containment of virus replication evidenced prolonged disease-free survival.

These findings suggest that available vaccine modalities that elicit potent CTL responses may be able to confer protection against the persistent high levels of viral replication routinely observed in HIV-infected individuals. Such vaccine-elicited immunity may slow the progression of disease in a vaccinated individual who subsequently is infected with HIV. A second potential benefit might also occur as a result of such a vaccine strategy. Vaccinated individuals who subsequently become infected with HIV may have low levels of replication-competent virus in their secretions. Since the likelihood of transmitting virus from an infected to an uninfected individual is correlated with the levels of virus in that infected individual's blood and secretions (26), such vaccinated and then infected individuals may be less likely to transmit virus than those who were infected without the benefit of prior vaccination. Thus, a CTL-inducing vaccine may ultimately slow the spread of HIV in the population. Vaccines that elicit immunity that attenuates disease and slows virus spread in a population may therefore be achievable. The potential benefits of such an outcome could be enormous, particularly in regions of the world where the absence of a medical infrastructure precludes the distribution of effective antiviral drugs to HIV-infected individuals.

However, a recent observation in nonhuman primates has suggested a likely limitation of a vaccine approach based solely on the elicitation of CTLs. In a monkey that was vaccinated and subsequently infected with an AIDS virus isolate, impressive early CTL control of replicating virus and clinical protection were lost as the virus accumulated mutations that allowed it to escape from CTL recognition (27). Viral escape from CTLs may therefore prove a general mechanism of vaccine failure in individuals receiving vaccines of this type. Nevertheless, such virologic events should occur infrequently if vaccine-elicited immune responses restrict viral replication to very low levels, since virus replicating at low levels would be expected to accumulate mutations relatively slowly. 


\section{Conclusions}

Although studies in nonhuman primates have shown that neutralizing antibodies can protect against infection by an AIDS virus, vaccine strategies for eliciting such immune responses remain elusive. With accumulating evidence for the importance of CTLs in containing HIV spread in an infected individual, a number of vaccine strategies are being pursued for elicitation of these immune effector cells. These strategies include the use of plasmid DNA, live recombinant viral vectors, and combined modality or prime/boost approaches. Interestingly, while these CTL-inducing vaccines have not elicited immunity that prevents AIDS virus infections in monkeys, they have generated immunity that contributes to containment of virus replication subsequent to infection. This containment of virus replication results in prolonged disease-free survival in vaccinated and then challenged monkeys. Early-phase testing of these novel vaccine strategies is ongoing in human volunteer populations. The conviction is growing that an HIV vaccine that at least slows disease progression, if not one that prevents infection, is now possible.

1. Malim, M.H., and Emerman, M. 2001. HIV-1 sequence variation: drift, shift, and attenuation. Cell. 104:469-472.

2. Hirsch, V.M., and Johnson, P.R. 1994. Pathogenic diversity of simian immunodeficiency viruses. Virus Res. 32:183-203.

3. Reimann, K.A., et al. 1996. A chimeric simian/human immunodeficiency virus expressing a primary patient human immunodeficiency virus type 1 isolate env causes an AIDS-like disease after in vivo passage in rhesus monkeys. J. Virol. 70:6922-6928.

4. Mascola, J.R., et al. 2000. Protection of macaques against vaginal transmission of a pathogenic HIV-1/SIV chimeric virus by passive infusion of neutralizing antibodies. Nat. Med. 6:207-210.

5. Walker, C.M., Moody, D.J., Stites, D.P., and Levy, J.A. 1986. CD8+ lymphocytes can control HIV infection in vitro by suppressing virus replication. Science. 234:1563-1566

6. Koup, R.A., et al. 1994. Temporal association of cellular immune responses with the initial control of viremia in primary human immunodeficiency virus type 1 syndrome. J. Virol. 68:4650-4655.

7. Ogg, G.S., et al. 1998. Quantitation of HIV-1-specific cytotoxic T lymphocytes and plasma load of viral RNA. Science. 279:2103-2106.

8. Altman, J.D., et al. 1996. Phenotypic analysis of antigen-specific T lymphocytes. Science. 274:94-96.
9. Schmitz, J.E., et al. 1999. Control of viremia in simian immunodeficiency virus infection by CD8+ lymphocytes. Science. 283:857-860.

10. Rosenberg, E.S., et al. 1997. Vigorous HIV-1-specific CD4+ $\mathrm{T}$ cell responses associated with control of viremia. Science. 278:1447-1450.

11. Daniel, M.D., Kirchhoff, F., Czajak, S.C., Sehgal, P.K., and Desrosiers, R.C. 1992. Protective effects of a live attenuated SIV vaccine with a deletion in the nef gene. Science. 258:1938-1941.

12. Baba, T.W., et al. 1995. Pathogenicity of live, attenuated SIV after mucosal infection of neonatal macaques. Science. 267:1820-1825.

13. Murphey-Corb, M., et al. 1989. A formalin-inactivated whole SIV vaccine confers protection in macaques. Science. 246:1293-1297.

14. Stott, E.J. 1991. Anti-cell antibody in macaques. Nature. 353:393.

15. Levine, A.M., et al. 1996. Initial studies on active immunization of HIVinfected subjects using a gp120-depleted HIV-1 immunogen: long-term follow-up. J. Acquir. Immune Defic. Syndr. 11:351-364.

16. Berman, P.W., et al. 1990. Protection of chimpanzees from infection by HIV-1 after vaccination with recombinant glycoprotein gp120 but not gp160. Nature. 345:622-625.

17. Egan, M.A., et al. 2000. Simian immunodeficiency virus (SIV) gag DNAvaccinated rhesus monkeys develop secondary cytotoxic T-lymphocyte responses and control viral replication after pathogenic SIV infection. J. Virol. 74:7485-7495

18. Amara, R.R., et al. 2001. Control of a mucosal challenge and prevention of clinical AIDS in rhesus monkeys by a multiprotein DNA/MVA vaccine. Science. 292:69-74.

19. Shen, L., et al. 1991. Recombinant virus vaccine-induced SIV-specific CD8+ cytotoxic T lymphocytes. Science. 252:440-443.

20. Redfield, R.R., et al. 1987. Disseminated vaccinia in a military recruit with human immunodeficiency virus (HIV) disease. N. Engl. J. Med. 316:673-676.

21. Evans, T.G., et al. 1999. A canarypox vaccine expressing multiple human immunodeficiency virus type 1 genes given alone or with rgp120 elicits broad and durable CD8+ cytotoxic T lymphocyte responses in seronegative volunteers. J. Infect. Dis. 180:290-298.

22. Shiver, J.W., et al. 2002. Replication-incompetent adenoviral vaccine vector elicits effective anti-immunodeficiency-virus immunity. Nature. 415:331-335.

23. Ourmanov, I., et al. 2000. Comparative efficacy of recombinant modified vaccinia virus Ankara expressing simian immunodeficiency virus (SIV) Gag-Pol and/or Env in macaques challenged with pathogenic SIV. J. Virol. 74:2740-2751.

24. Barouch, D.H., et al. 2000. Control of viremia and prevention of clinical AIDS in rhesus monkeys by cytokine-augmented DNA vaccination. Science. 290:486-492.

25. Barouch, D.H., et al. 2001. Reduction of simian-human immunodeficiency virus $89.6 \mathrm{P}$ viremia in rhesus monkeys by recombinant modified vaccinia virus Ankara vaccination. J. Virol. 75:5151-5158.

26. Quinn, T.C., et al. 2000. Viral load and heterosexual transmission of human immunodeficiency virus type 1. N. Engl. J. Med. 342:921-929.

27. Barouch, D.H., et al. 2002. Eventual AIDS vaccine failure in a rhesus monkey by viral escape from cytotoxic $\mathrm{T}$ lymphocytes. Nature. 415:335-339. 